\title{
Need for standardized endoscopic tattooing agents
}

\author{
Prachi S Patil • Avanish Saklani • Shaesta Mehta
}

Published online: 6 November 2013

(C) Indian Society of Gastroenterology 2013

Sir,

India ink tattooing has been used to mark lesions that can be identified subsequently at the time of surgery or endoscopy. We recently attempted endoscopic tattooing of two patients with colorectal tumor/polyps who were planned for surgery and would like to share our experience regarding the same. Patient "A" had a transverse colon tumor with a few scattered polyps in the transverse and the right colon. Patient " $\mathrm{B}$ " had a rectosigmoid tumor with a polyp at $12 \mathrm{~cm}$ from the anal verge. Both patients underwent a complete colonoscopy after a bowel preparation followed by a 4-quadrant tattooing of the lesion (transverse colon tumor and most distal polyp in patient " $\mathrm{A}$ " and rectal polyp in patient " $B$ "). We used non-sterile India ink (1:100 dilution with normal saline) which was rendered sterile by autoclaving at $121^{\circ}$ for $35 \mathrm{~min}$. At the time of surgery (performed 3 days after colonoscopic tattooing in patient " $\mathrm{A}$ " and 4 days in patient "B"), in both patients, the tattoo could not be identified and an intraoperative endoscopy was required in patient "B" for lesion localization. In this patient, mild staining was identified around the polyp after cutting open the specimen.

Endoscopic tattooing was first described more than 30 years ago [1]. India ink is the most commonly used agent for tattooing. Other agents include methylene blue, indigo carmine, and indocyanine green (ICG). Most dyes are reabsorbed within $24 \mathrm{~h}$ except for India ink and ICG [2]. A sterile carbonbased permanent marker formulation called SPOT ${ }^{\mathrm{TM}}$ is

P. S. Patil $(\triangle) \cdot$ S. Mehta

Department of Digestive Diseases and Clinical Nutrition, Tata

Memorial Hospital, Dr Ernest Borges Road, Parel, Mumbai 400 012,

India

e-mail: prachipatil@gmail.com

\section{A. Saklani}

Division of Colorectal Surgery, Department of Surgical Oncology,

Tata Memorial Hospital, Dr Ernest Borges Road, Parel, Mumbai 400

012, India licensed for endoscopic tattooing abroad and is considered to be safe and effective [3]. SPOT ${ }^{\mathrm{TM}}$ is however unavailable in India. The ASGE technology status evaluation report recommends sterilization of non-sterile India ink by autoclaving or passing through a Millipore filter [2].

We used commercially available non-sterile India ink and then diluted it and autoclaved it as per the ASGE recommendation. We are unsure about whether autoclaving India ink has an adverse effect on the durability of the tattoo. Conversely, a higher concentration of India ink may be needed if it is to be autoclaved. There is an unmet need of standardized and approved tattooing agents in India.

Laparoscopic colorectal surgery as well as robotic colorectal surgery is getting popular in India. There is an urgent need for making FDA-approved tattooing agents like SPOT ${ }^{\mathrm{TM}}$ available in India.

\section{References}

1. Ponsky JL, King JF. Endoscopic marking of colonic lesions. Gastrointest Endosc. 1975;22:42-3.

2. ASGE Technology Committee, Kethu SR, Banerjee S, Desilets D, et al. Endoscopic tattooing. Gastrointest Endosc. 2010;72:681-5.

3. Askin MP, Waye JD, Fiedler L, et al. Tattoo of colonic neoplasms in 113 patients with a new sterile carbon compound. Gastrointest Endosc. 2002;56:339-42. 